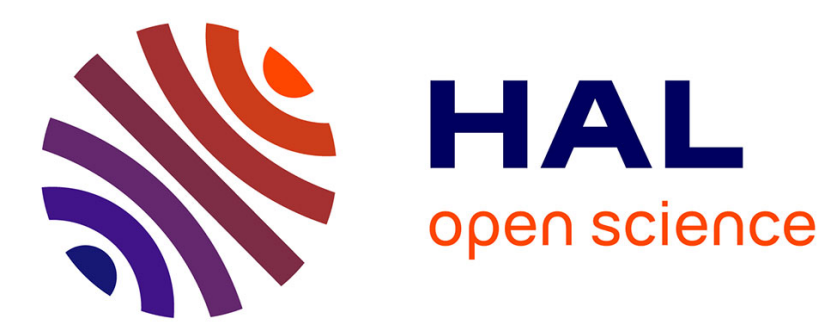

\title{
Delaying transition to turbulence by a passive mechanism
}

\author{
J.H.M. Fransson, A. Talamelli, L. Brandt, Carlo Cossu
}

\section{To cite this version:}

J.H.M. Fransson, A. Talamelli, L. Brandt, Carlo Cossu. Delaying transition to turbulence by a passive mechanism. Physical Review Letters, 2006, 96 (6), pp.064501. 10.1103/physrevlett.96.064501 . hal01023352

\section{HAL Id: hal-01023352 \\ https://hal-polytechnique.archives-ouvertes.fr/hal-01023352}

Submitted on $20 \mathrm{Jul} 2014$

HAL is a multi-disciplinary open access archive for the deposit and dissemination of scientific research documents, whether they are published or not. The documents may come from teaching and research institutions in France or abroad, or from public or private research centers.
L'archive ouverte pluridisciplinaire HAL, est destinée au dépôt et à la diffusion de documents scientifiques de niveau recherche, publiés ou non, émanant des établissements d'enseignement et de recherche français ou étrangers, des laboratoires publics ou privés. 


\title{
Delaying Transition to Turbulence by a Passive Mechanism
}

\author{
Jens H. M. Fransson, ${ }^{1, *}$ Alessandro Talamelli, ${ }^{2,1}$ Luca Brandt, ${ }^{1}$ and Carlo Cossu ${ }^{3}$ \\ ${ }^{1}$ KTH Mechanics, SE-10044, Stockholm, Sweden \\ ${ }^{2}$ II Facoltà di Ingegneria, Università di Bologna, I-47100 Forlì, Italy \\ ${ }^{3}$ LadHyX, CNRS Ecole Polytechnique, F-91128 Palaiseau, France \\ (Received 21 July 2005; published 17 February 2006)
}

\begin{abstract}
Reducing skin friction is important in nature and in many technological applications. This reduction may be achieved by reducing stresses in turbulent boundary layers, for instance tailoring biomimetic rough skins. Here we take a second approach consisting of keeping the boundary layer laminar as long as possible by forcing small optimal perturbations. Because of the highly non-normal nature of the underlying linearized operator, these perturbations are highly amplified and able to modify the mean velocity profiles at leading order. We report results of wind-tunnel experiments in which we implement this concept by using suitably designed roughness elements placed on the skin to enforce nearly optimal perturbations. We show that by using this passive control technique it is possible to sensibly delay transition to turbulence.
\end{abstract}

For streamlined bodies moving in fluids the viscous drag is generated in the thin boundary layer surrounding the body skin, where the velocity of the fluid decreases to zero at the surface. In the upstream part of a streamlined body, the boundary layer flow is laminar: its velocity profiles are steady and stable to the small perturbations introduced by imperfections of the skin, turbulence in the incoming flow, and other noise sources. It is, however, well known that sufficiently far downstream (where the Reynolds number $\operatorname{Re}=U_{\infty} x / \nu$ based on the incoming velocity $U_{\infty}$, the downstream distance from the leading edge $x$ and the kinematic viscosity $\nu$ of the fluid, exceeds a critical value) the flow becomes unstable and small perturbations can be amplified, leading to transition to turbulence when they exceed a critical finite amplitude [1]. Owing to the increased shear stresses the mean skin friction of a turbulent boundary layer is typically an order of magnitude larger than that of a laminar flow. In the turbulent regime, drag may be reduced by different means [2-5], not rarely inspired by nature and its evolution $[2,6]$. An alternative approach to reduce the viscous drag is to shift the transition point downstream [7]. The simplest configuration used to test transition prediction and control tools is the boundary layer on a flat plate. In this case, the laminar boundary layer flow is given by the family of Falkner-Skan self-similar solutions and the unstable waves are the so called Tollmien-Schlichting (TS) waves [8].

Recent numerical simulations [9] and stability analysis [10] predicted that narrow spanwise modulations of the boundary layer thickness, the so called streaks, can have a stabilizing effect on low amplitude TS waves. Boundary layer streaks can be very efficiently generated by inserting streamwise vortices near the leading edge of the flat plate. If these vortices are of "optimal" shape, their energy can be amplified of $O\left(\operatorname{Re}^{2}\right)$ through the lift-up effect $[11,12]$. This effect exploits the strong non-normality of the under- lying stability operator [13]. Recent wind-tunnel experiments have shown that steady and stable streaks of moderate amplitude can be induced by generating nearly optimal vortices with cylindrical roughness elements placed near the leading edge [14], and proved that these streaks have a stabilizing effect on the TS waves [15]. However, secondary instabilities and the final breakdown to turbulence set in when the Tollmien-Schlichting waves reach amplitudes larger than those previously investigated. At finite amplitudes, TS waves are likely to nonlinearly interact with the streaks and the impact of this interaction on the stability of the boundary layer is still partially unclear. Boiko et al. [16] found that, in the presence of unsteady and randomly distributed streaks, TS waves are stabilized in the mean when they are of small amplitude. However, when forced at larger amplitudes, they promote transition. In the case of the nonlinear interactions of TS waves and streaks associated to Görtler vortices, which arise as a primary instability in boundary layers on concave surfaces, theoretical [17,18] and numerical [19] analyses predict a destabilizing effect. An additional matter of concern is that, as it is well known, if the streaks reach a critical threshold amplitude, they become unstable to high frequency waves characterized by growth rates 1 order of magnitude larger than those of the TS waves. These initiate a breakdown to turbulence, usually referred to as "bypass" $[20,21]$, which is more likely to occur in the presence of high levels of the external perturbation. It is therefore not currently clear whether the stabilizing action of the streaks on the small amplitude TS waves can be effectively used to delay transition in the boundary layer. Transition to turbulence is indeed an inherently nonlinear phenomenon and one should not only consider the laminar basic flow but other nontrivial attractors or repellors of the whole system [22]. As recently shown, streaks play an important role in the formation of these states and it can therefore be argued 
that by forcing them the flow is brought closer to the nontrivial states. Here, instead of embarking in a theoretical and/or numerical stability analysis of the nonlinear interactions between finite amplitude TS waves and streaks, with all the necessary but restricting simplifying assumptions needed to obtain some tractable cases, we decided to directly experimentally ascertain whether any transition delay is observed when streaks are forced in a transitional boundary layer. The aim of this investigation is therefore to prove that controlled low energy optimal perturbations can, due to their large transient amplification in a non-normal system, effectively stabilize that system even in the nonlinear regime and can indeed be used for control purposes. Such a conclusion would be of interest in applications where the importance of non-normality and transient growth has been recognized, such as lasers [23], ionosphere's dynamics [24], magnetohydrodynamics [25], plasma physics [26,27], granular flow dynamics [28], and thermocapillary driven spreading [29] to cite only some.

The boundary layer flow under investigation is forming on a flat plate mounted horizontally in the test section of the minimum-turbulence-level (MTL) wind-tunnel at KTH Mechanics. The dimensions of the test section are $7.0 \times$ $0.8 \times 1.2 \mathrm{~m}^{3}$ and the flow is subject to a weak adverse pressure gradient (by adjusting the ceiling) which enhances the instability and thus favors transition. For a more thorough description of the wind-tunnel and experimental setup see Ref. [15]. A free-stream velocity at the leading edge of $U_{\infty}=5.0 \mathrm{~ms}^{-1}$ was kept constant throughout all experiments. Owing to the convective nature of boundary layer instabilities, the transition location strongly depends on the type and level of environmental noise. In the present configuration natural (unforced) transition occurs far downstream, outside the measurement region. Therefore to obtain a well-defined and reproducible transition location, disturbances were forced in a controlled way following the classical approach [30-32]. As in a previous experiment [15], TS waves were introduced by unsteady blowing and suction at the wall through a slot in a plug (Fig. 1). The sinusoidal signal is computer generated through a $D / A$ board to an audio amplifier driving two loud speakers, which were connected to the slot through vinyl hoses. A frequency $(f)$ of $32 \mathrm{~Hz}$ has been selected so that transition could be triggered within the observation and measurement regions after the exponential growth of the TS waves. The amplitude of the signal to the loud speakers was quantified by measuring the ac output voltage from the amplifier with a voltage meter.

To visualize the flow state, smoke is injected through a second slot (see Fig. 1). A CCD camera $(1280 \times$ 1024 pixels) is used to record images in an area of $210 \times$ $168 \mathrm{~mm}^{2}$ located $1434 \mathrm{~mm}$ downstream of the leading edge (Fig. 1). Without forcing, the two-dimensional laminar flow is observed [Fig. 2(a)], whereas forcing with the amplifier set at $201 \mathrm{mV}$ triggers a turbulent flow in the observation window [Fig. 2(b)]. The same experiment is

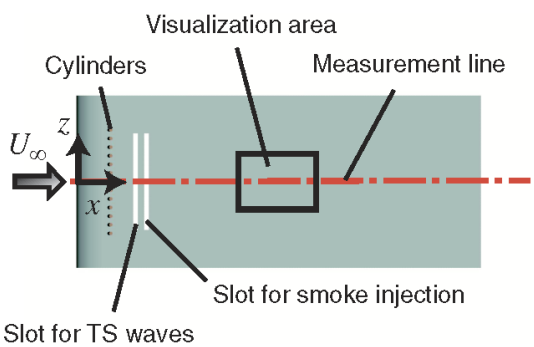

FIG. 1 (color online). Plane view of the plate used in the experiment. Forty-two cylindrical roughness elements $($ diameter $=4.2 \mathrm{~mm}$ and height $=1.4 \mathrm{~mm}$ ) are positioned $80 \mathrm{~mm}$ from the leading edge and periodically spaced in the spanwise direction by $14.7 \mathrm{~mm}$. The slot for the TS generation, followed by the one for the smoke injection $(330 \mathrm{~mm}$ long in the spanwise direction and $0.8 \mathrm{~mm}$ wide) are located at $x=190$ and $213 \mathrm{~mm}$, respectively. A CCD camera $(1280 \times 1024$ pixels $)$ is used to record the images in an area of $210 \times 168 \mathrm{~mm}^{2}$ located at $x=1434 \mathrm{~mm}$.

then repeated in the spanwise modulated boundary layer flow. The modulation is obtained as in Ref. [15], i.e., by pasting on the flat plate 42 cylindrical roughness elements (diameter $=4.2 \mathrm{~mm}$ and height $=1.4 \mathrm{~mm}$ ) periodically spaced in the spanwise direction by $14.7 \mathrm{~mm}$ and at a

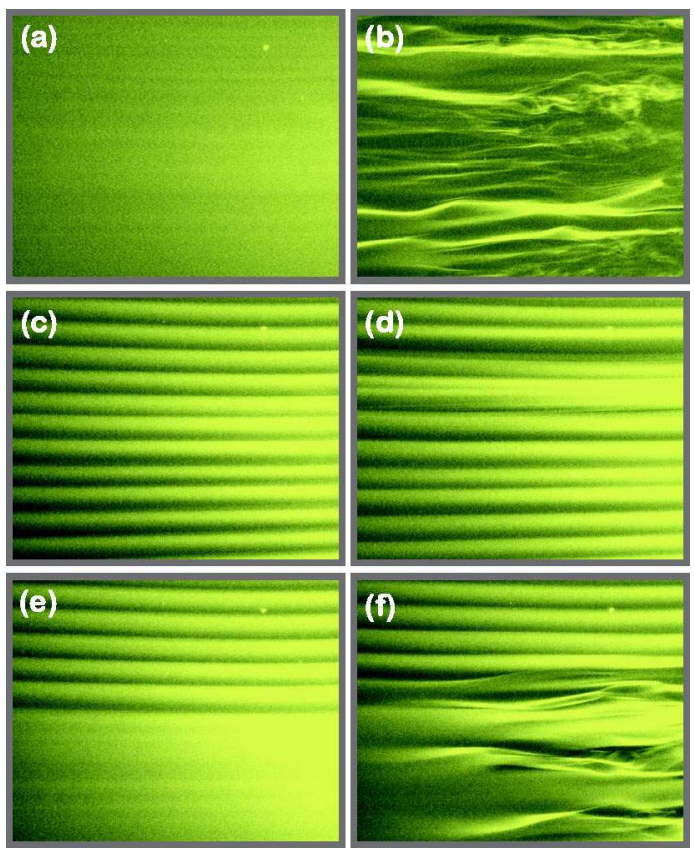

FIG. 2 (color online). Smoke flow visualizations from above with flow from left to right. (a) and (b) show the two-dimensional boundary layer, without streaks, with no excitation and with excitation of $201 \mathrm{mV}$, respectively. The flow in (b) is turbulent, (c) shows the streaky base flow with no excitation. In the presence of streaks with excitation of $450 \mathrm{mV}$ (d), the flow remains laminar; (e) shows a half-streaky boundary layer obtained removing half the roughness elements and without forcing. With a forcing at $157 \mathrm{mV}$ (f), the streaky part of the boundary layer remains laminar while the uncontrolled part undergoes transition. 
distance of $80 \mathrm{~mm}$ from the leading edge. Here it should be emphasized that the net drag increase due to the presence of the streaks is less than $3.5 \%$ compared to the $2 \mathrm{D}$ base flow. This estimation is based on a momentum balance analysis, performed on a similar streaky base flow, using 30 wall-normal velocity profiles per period of the streaks in the spanwise direction and at five different downstream positions in the interval 19-572 roughness heights or $\mathrm{Re} \approx$ $(18-167) \times 10^{3}$. The streaky base flow induced by the roughness is shown in Fig. 2(c). When subject to the same disturbance forcing as before this streaky flow remains laminar in the visualization region. Even when the excitation amplitude is doubled to $450 \mathrm{mV}$, as shown in Fig. 2(d), the flow is kept laminar. The experiment has then been repeated removing the roughness elements on one half of the plate. The half-streaky basic flow obtained in the absence of disturbance forcing is shown in Fig. 2(e), while in Fig. 2(f) we observe a forced case with an amplitude of $157 \mathrm{mV}$. It is clear from the figures that the modulated boundary layer is laminar while the two-dimensional flow is transitional.

Quantitative data have been obtained by hot-wire measurements of the streamwise velocity component $U$ along the plate at constant dimensionless height, $y / \delta=2.2$, where $\delta=\left(x \nu / U_{\infty}\right)^{1 / 2}$ measures the boundary layer thickness. To force three-dimensional disturbances, a classical procedure was used [33], i.e., thin $50 \times 50 \mathrm{~mm}^{2}$ tape spacers were pasted on the flat plate just downstream of the blowing/suction slot. The streamwise distribution of the temporal root mean square (rms) of the velocity fluctuations and two typical hot-wire time signals, induced by a forcing of $1000 \mathrm{mV}$, are reported in Fig. 3. In the absence of streaks the classical $K$-type transition scenario is observed [33,34]. Linear TS waves [Fig. 3(b)] of small amplitude evolve into finite amplitude nonlinear waves, characterized by the appearance of spikes in the hot-wire traces. This leads to an increase of the rms velocity to a peak level [33] where the flow is transitional. Transition is completed when the perturbation rms velocity reaches a plateau $(x=1650 \mathrm{~mm})$ where a fully turbulent signal is observed [Fig. 3(c)]. In the presence of streaks the flow is laminar: the forced perturbations remain of small amplitude and sinusoidal all along the measurement region as it can be seen comparing the signals with and without streaks at $x=1650 \mathrm{~mm}$ in Fig. 3(c). Thus, we show that it is possible, using the streaks, to maintain TS waves at low amplitudes in a boundary layer flow which otherwise would be transitional in the absence of the streaks. Increasing the excitation amplitudes of the TS waves, transition is observed also in the streaky boundary layer, even though further downstream than in the twodimensional boundary layer and no delay is observed for forcing amplitudes larger than $2000 \mathrm{mV}$. Transition is never observed to occur earlier in the streaky flow than in its two-dimensional counterpart. These results are in accordance with the observation in Ref. [16] that forcing TS

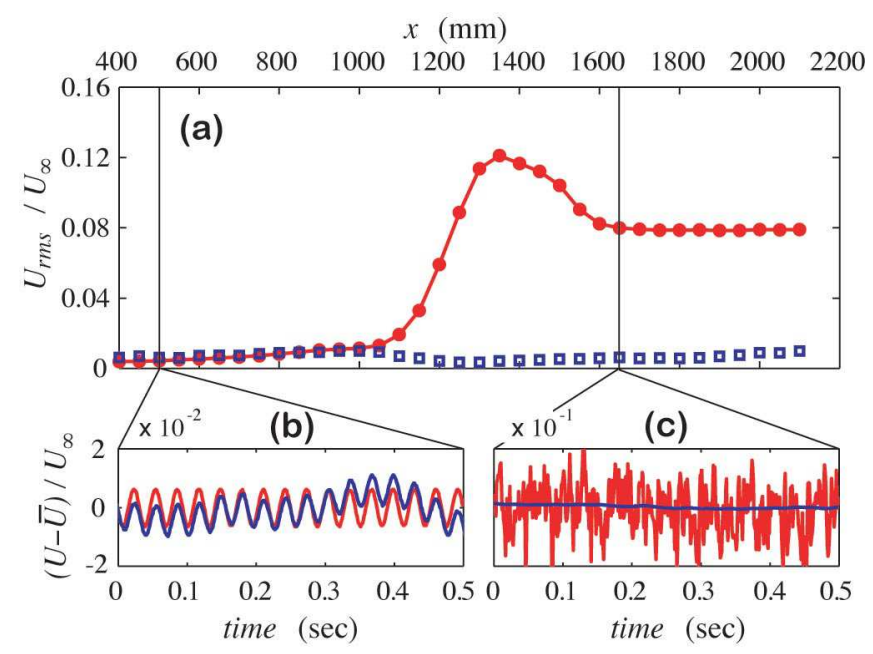

FIG. 3 (color online). (a) Evolution of the dimensionless temporal root mean square streamwise velocity fluctuations $U_{\text {rms }} / U_{\infty}$ in the streamwise direction $x$ when TS waves are excited at $f=32 \mathrm{~Hz}$ and $1000 \mathrm{mV}$. (b),(c) The time signals at two selected positions ( $x=400$ and $x=1650 \mathrm{~mm}$ ). Light (red) lines and solid (red) symbols correspond to the two-dimensional boundary layer, without streaks, whereas dark (blue) lines and open (blue) symbols to the boundary layer with the controlling streaks present. In the presence of streaks the flow remains laminar.

waves with high enough amplitudes into a streaky boundary layer transition is promoted. These findings also imply that in the present configuration there is no significant destabilizing action due to the nonlinear interactions between streaks and TS waves and therefore the low amplitude stabilizing action of the streaks on the TS waves is able to delay transition. The results are essentially unchanged when random noise is added to the forcing. Transition delay is also observed when white noise with amplitude of $10 \%$ of that of the periodic signal is added in the excitation signal, showing that the delay mechanism is robust for strongly colored noise. Experiments were also performed by forcing large amplitude $2000 \mathrm{mV}$ white noise without the sinusoidal signal. TS waves of all frequencies are thus excited with random phase shifts. In Fig. 4(a) we show the streamwise evolution of the temporal root mean square of the perturbation whereas in Fig. 4(b) the intermittency factor $\gamma$ is displayed. The latter measures the fraction of turbulent signal in the hot-wire trace [35], and a value of $\gamma=0.5$ is often used as a measure of transition location whereas $\gamma=0.1$ is used to determine the transition onset. Even in this case, the streaks delay transition (385 mm delay of the location where $\gamma=0.1$ and $370 \mathrm{~mm}$ delay of the $\gamma=0.5$ position). In Fig. 4 one may also observe that the rms-velocity peak in the streamwise direction corresponds [35] to an intermittency factor of about 0.5 .

In conclusion, we have shown that transition to turbulence in a boundary layer can be delayed by a passive method. Thus, contrary to the common belief of fluid 

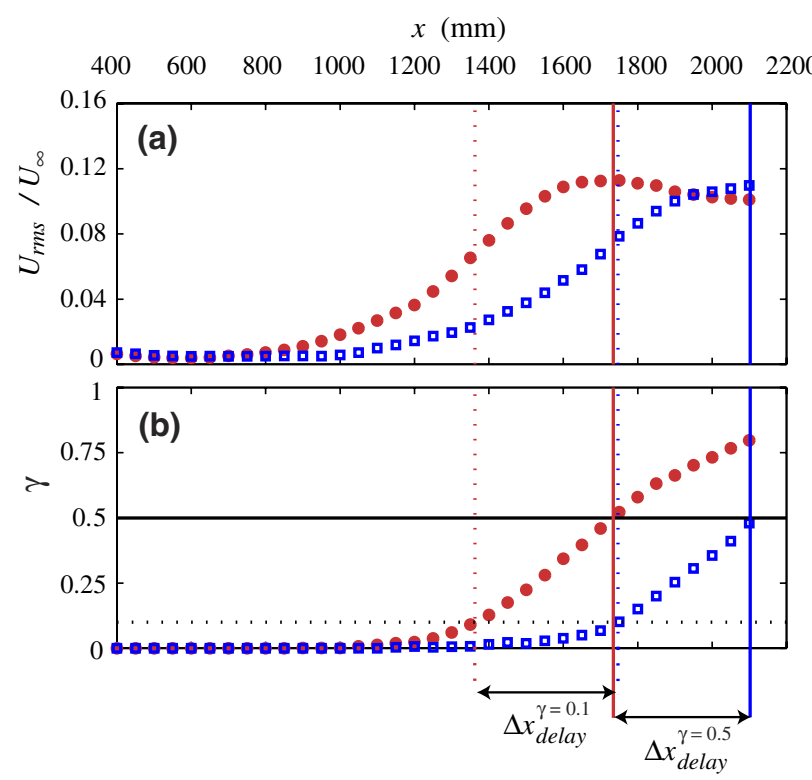

FIG. 4 (color online). (a) Dimensionless temporal root mean square of the streamwise velocity fluctuations $U_{\text {rms }} / U_{\infty}$ and (b) intermittency factor $\gamma$ distributions in the streamwise direction $x$ when a random (white noise) instead of sinusoidal (TS) excitation is used. Solid (red) symbols correspond to the boundary layer without streaks and open (blue) symbols to the boundary layer with the controlling streaks present. In the presence of streaks, the transition is delayed as indicated by the downstream shift $\Delta x_{\text {delay }}$ of the locations where the levels $\gamma=0.1$ and $\gamma=0.5$ are attained.

dynamicists, sometimes roughness, if well designed, can reduce the viscous drag. The observed delay can be ascribed to the reduction of the exponential growth of TS waves evolving in boundary layer flows in the presence of streaks, and to the absence of strongly destabilizing nonlinear interaction between these two types of perturbation.

The authors wish to thank P. H. Alfredsson for his valuable suggestions during the experiments and for critically reading the manuscript. M. Matsubara is acknowledged for his valuable suggestions regarding the flow visualization technique and P. J. Schmid for very fruitful discussions. M. Gällstedt is acknowledged for his assistance in the manufacturing of the roughness elements.

*To whom correspondence should be addressed. Electronic address: jensf@mech.kth.se

[1] T. Herbert, Annu. Rev. Fluid Mech. 20, 487 (1988).

[2] D. W. Bechert, M. Bruse, W. Hage, J. G. T. V. D. Hoeven, and G. Hoppe, J. Fluid Mech. 338, 59 (1997).

[3] L. Sirovich and S. Karlsson, Nature (London) 388, 753 (1997).
[4] Y. Du and G. E. Karniadakis, Science 288, 1230 (2000).

[5] N. S. Bearman, Annu. Rev. Fluid Mech. 10, 47 (1978).

[6] D. M. Bushnell and K. J. Moore, Annu. Rev. Fluid Mech. 23, 65 (1991).

[7] R. D. Joslin, Annu. Rev. Fluid Mech. 30, 1 (1998).

[8] H. Schlichting, Boundary-Layer Theory (Mc Graw-Hill, New York, 1979).

[9] C. Cossu and L. Brandt, Phys. Fluids 14, L57 (2002).

[10] C. Cossu and L. Brandt, Eur. J. Mech. B, Fluids 23, 815 (2004).

[11] M. T. Landahl, J. Fluid Mech. 98, 243 (1980).

[12] L. H. Gustavsson, J. Fluid Mech. 224, 241 (1991).

[13] L. N. Trefethen, A. E. Trefethen, S. C. Reddy, and T. A. Driscoll, Science 261, 578 (1993).

[14] J. H. M. Fransson, L. Brandt, A. Talamelli, and C. Cossu, Phys. Fluids 16, 3627 (2004).

[15] J. H. M. Fransson, L. Brandt, A. Talamelli, and C. Cossu, Phys. Fluids 17, 054110 (2005).

[16] A. V. Boiko, K. J. A. Westin, B. G. B. Klingmann, V. V. Kozlov, and P. H. Alfredsson, J. Fluid Mech. 281, 219 (1994).

[17] A. H. Nayfeh, J. Fluid Mech. 107, 441 (1981).

[18] A. H. Nayfeh and A. Al-Maitaah, Phys. Fluids 31, 3543 (1988).

[19] M. R. Malik, T. A. Zang, and M. Y. Hussaini, J. Comp. Physiol. 61, 64 (1985).

[20] M. V. Morkovin, in Viscous Drag Reduction, edited by C. S. Wells (Plenum, New York, 1969).

[21] M. Matsubara and P. H. Alfredsson, J. Fluid Mech. 430, 149 (2001).

[22] B. Hof, C. W. H. van Doorne, J. Westerweel, F. T. M. Nieuwstadt, H. Faisst, B. Eckhardt, H. Wedin, R. Kerswell, and F. Waleffe, Science 305, 1594 (2004).

[23] H. J. Landau, J. Opt. Soc. Am. 66, 525 (1976).

[24] J.P. Flaherty, C.E. Seyler, and L.N. Trefethen, J. Geophys. Res. 104, 6843 (1999).

[25] B. F. Farrell and P. J. Ioannou, Astrophys. J. 522, 1079 (1999).

[26] F. Volponi, Z. Yoshida, and T. Tatsuno, Phys. Plasmas 7, 2314 (2000).

[27] T. Tatsuno, F. Volponi, and Z. Yoshida, Phys. Plasmas 8, 399 (2001).

[28] P. J. Schmid and H. K. Kytomaa, J. Fluid Mech. 264, 255 (1994).

[29] J. M. Davis and S. M. Troian, Phys. Rev. E 70, 046309 (2004).

[30] G. B. Schubauer and H. K. Skramstad, J. Res. Natl. Bur. Stand. (U.S.) 38, 251 (1948).

[31] G. B. Schubauer and P. S. Klebanoff, Natl. Advis. Comm. Aeron. Tech. Note (1955).

[32] I. Tani, Annu. Rev. Fluid Mech. 1, 169 (1969).

[33] P. S. Klebanoff, K. D. Tidstrom, and L. M. Sargent, J. Fluid Mech. 12, 1 (1962).

[34] Y. S. Kachanov, Annu. Rev. Fluid Mech. 26, 411 (1994).

[35] J.H. M. Fransson, M. Matsubara, and P.H. Alfredsson, J. Fluid Mech. 527, 1 (2005). 